\title{
As a black start the wind power storage system has a storage capacity configuration
}

\author{
HU Haiming ${ }^{1, *}$, Yan Yan $^{1}$ \\ ${ }^{1}$ Shanghai Dianji University, Lingang, Pudong New Area, Shanghai, China
}

\begin{abstract}
Through multiple simulations and statistics of its capacity deficit data, the basic value of the energy storage capacity that can cover most wind conditions is determined. Considering that during the black start process, other aspects other than the plant electricity also occupy the energy storage capacity, and the capacity compensation is made in the calculation. Finally, the verification model shows that its energy storage capacity configuration can support the black start task of the auxiliary plant in the area under most wind conditions and has certain economic efficiency.
\end{abstract}

\section{Introduction}

In an environment where microgrids are becoming more and more popular, it is of great significance to consider using wind storage systems as black start power sources for power plants. Wind power output fluctuates greatly, but energy storage technology can achieve smooth wind farm output in wind power grid connection, ensure continuous and reliable power supply of wind power generation system, and improve system operation reliability and redundancy. Energy storage plays an irreplaceable role in achieving stable power quality output and power support for wind power.

Energy storage capacity configuration is usually divided into two main categories, namely theoretical analysis and simulation methods. The theoretical analysis method generally analyzes the volatility of wind power based on historical data and extracts features, and then mathematically models the power and capacity of energy storage based on the features of power fluctuations. For example, literature [1] established a wind farm energy storage system capacity optimization model, and used particle swarm algorithm to solve the optimization model. Literature [2] proposed a scale calculation method between different energy storage scales under technical and economic indicators based on the analytic hierarchy process. Literature [3] established the basic output calculation model of the microgrid group, and carried out the corresponding cost analysis, and elaborated the principle and calculation process of the microgrid energy storage optimization configuration based on the improved biogeographic optimization algorithm. The configuration method of simulation analysis is generally based on a large number of or typical wind power data, using the existing wave suppression control and energy management methods to simulate and analyze the suppression control effect. Finally, the minimum energy storage power and capacity required under the premise of meeting the fluctuation limit index are obtained. For example, literature [4] uses computer technology and WT software to simulate the actual situation of the wind field to establish a calculation model, and calculate the optimal energy storage capacity from the direction of computational fluid dynamics. Literature [5] is based on the active output data of the energy storage system obtained from the simulation, considering the influence of the initial state of charge range, to minimize the cost of configuring the battery module capacity and maximizing the range of the initial state of charge to solve the optimal rated capacity. However, the article did not study the influence of wind speed fluctuations to optimize the configuration. This paper uses simulation analysis to optimize the configuration of energy storage combined with wind speed fluctuations.

\section{The wind storage system supports the black start architecture of thermal power plants}

Assuming that the initial state of the power plant is the hot state, the power demand of its auxiliary machinery and other plant equipment is about $12 \mathrm{MW}$ concerning the thermal state of a $300 \mathrm{MW}$ power plant. After all the auxiliary engines are started, it needs to go through the links of steam turbine speed regulation and generator gridconnected power generation, so the black start process will take about 3 hours. To realize the black start of the power plant, its wind storage system must provide power support for more than 3 hours.

Suppose that each wind power in the system is rectified by the inverter and then connected to a $0.96 \mathrm{KV} / 35 \mathrm{KV}$ box-type transformer and connected to the $35 \mathrm{KV}$ busbar of the wind power collection system. The bus of the wind power collection system is connected with the auxiliary power bus through a $35 \mathrm{KV} / 10 \mathrm{KV}$ transformer. The energy

*Corresponding author's E-mail: huhaiminga@21cn.com 
storage system is connected to the collector bus through the rectifier device to exchange energy with the outside world. Its basic structure is shown in Figure 1:

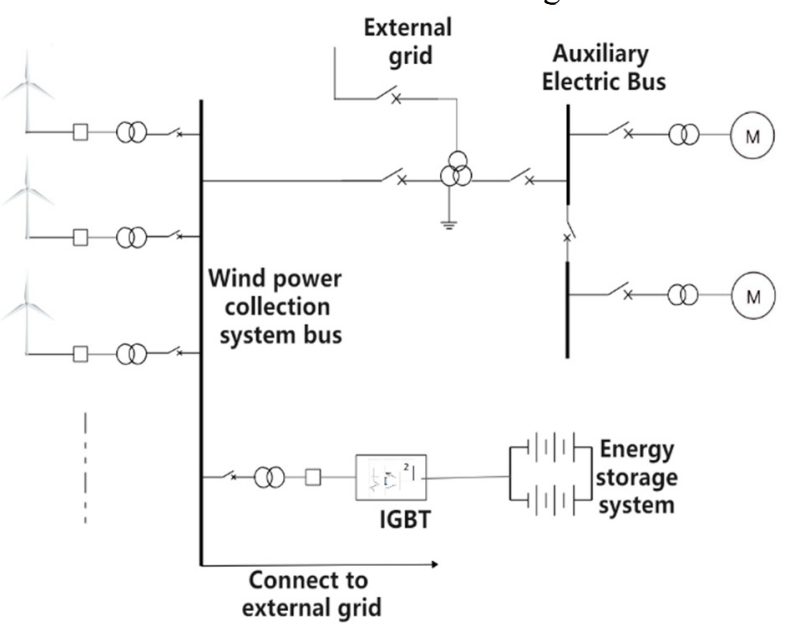

Figure 1 Basic architecture of black start

The number and capacity of wind power in the wind storage system are determined by the power requirements of the power plant's auxiliary machinery combined with the annual average wind speed environment of the wind farm. The average wind speed data in the statistical period can be obtained from the following formula, and the basic formula is as follows:

$$
V=\frac{1}{n} \sum_{i=1}^{n} v_{i}
$$

Where: $V$ is the average wind speed of the wind farm in the statistical period; $n$ is the number of representative wind measurement points in the field in the statistical period; $v_{i}$ is the average wind speed of the $i$-th wind measurement tower in the statistical period.

Based on the historical wind speed data of the wind farm, the annual average wind speed is counted, combined with the reference power of the wind power output model and its demand can be used to obtain the installed capacity of the wind power of the wind farm. According to the classic wind power model as equation (2), the power generated by single wind power at $\mathrm{V}$ wind speed can be obtained.

$$
\left\{\begin{array}{l}
p=0, v<v_{c i}, v>v_{c o} \\
p=p_{r} \frac{\left(v-v_{c i}\right)}{\left(v_{r}-v_{c i}\right)}, v_{c i}<v<v_{r} \\
p=p_{r}, \quad v_{r}<v<v_{c o}
\end{array}\right.
$$

In the formula: $V r$ and $P r$ respectively represent the rated wind speed and rated power of a single wind power; $v_{c i}$ and $v_{c o}$ are its cut-in and cut-out wind speeds.

The black start process usually lasts up to several hours. Due to changes in the environmental wind speed and the rapid increase in power demand during the start-up process of the auxiliary machinery of the power plant, the output of the wind farm may be insufficient. Therefore, it is considered that as the power demand increases in a short period of time, when the maximum power of the wind farm at the wind speed is reached, the excess power capacity is provided by the energy storage system to maintain the power balance.

\section{Energy storage system capacity configuration}

\subsection{Related parameters}

Energy storage capacity configuration indicators include power capacity configuration and energy capacity configuration. The energy storage power capacity configuration determines the ability to support the instantaneous power shortage of wind power to prevent the frequency or voltage drop caused by the insufficient power output of the wind power system. Energy storage energy capacity configuration affects the duration of energy storage power support. Both of whether storage can eliminate wind power shortfall due to the environmental impact caused by wind speed, voltage and frequency fluctuations and the cost of storage configuration whether the economy is important.

\subsection{Influencing factors of energy storage capacity}

Through the above analysis of the black start model and principle, the main factors that determine the configuration of energy storage capacity are as follows: (1) The magnitude of the wind speed fluctuation of the wind farm. The magnitude of wind speed fluctuations directly affects the stability of wind power output power, especially when the wind power of the wind farm continues to be too low and the wind power output is seriously insufficient, to maintain the orderly progress of the black start, it is necessary to fill the black start power gap from energy storage. (2) The energy storage power consumed by the self-starting of the wind farm needs to be compensated for the energy storage capacity. (3) The effect of the efficiency of energy storage batteries and inverter devices on the redundancy configuration of energy storage capacity.

\subsection{Capacity calculation}

\subsubsection{Black start supply and demand curve determination method}

To obtain the power output curve data close to the actual operation of wind power, it is necessary to import the time series wind speed of different periods into the simulation model for the black start simulation. According to the definition of time series, $\mathrm{x}_{1}, \mathrm{x}_{2}, \ldots, \mathrm{x}_{\mathrm{t}}$ are a time series, where " $x$ " is the wind speed data of the time series and " $t$ " is the length of time. Since the ultimate goal is to ensure the reliability of wind-assisted black start-up at a low investment, it is necessary to perform multiple simulations in different periods and count the data.

To improve the sampling efficiency of wind speed data samples, analyzing the seasonal index of the wind speed 
database can know the relationship between the season and the total average so that we can select the time series in a targeted manner. First, calculate the average of each period in the cycle to get the long-term average level of the period. Assume that the data structure of the sequence is $\mathrm{m}$ period (the month), and there are $\mathrm{n}$ periods (the same month) in total. The average for the same month is

$$
\bar{x}_{i}=\frac{\sum_{k=1}^{n} x_{i k}}{n}, i=1,2, \ldots 12
$$

Statistics of the total monthly average in the historical database:

$$
\bar{x}=\frac{\sum_{k=1}^{n} \sum_{i=1}^{12} x_{i k}}{n m}
$$

The average of the period divided by the total average can get the seasonal index of each period, as follows

$$
S_{i}=\frac{\bar{x}_{i}}{\bar{x}}, i=1,2, \ldots m
$$

If the seasonal index is greater than 1 , it means that the value of the month is higher than the average value, that is, the wind power output is sufficient in the month. If this ratio is less than 1 , it means that the monthly value is lower than the overall average. If the seasonal index is approximately equal to 1 , it means that there is no obvious seasonal effect in the month, and its value is usually close to the database average. In order to cover different seasonal characteristics more comprehensively and improve sampling efficiency, one month with the smallest seasonal index is selected as the time interval for sampling time series wind speed data. The grid fault disassembly can be considered as a random event, so the random sampling method is adopted for this seasonal interval to randomly select a time node in a time interval as the starting point of the time series wind speed. The total time for sampling the time series sample interval is 2400 hours, and the number of sampling can be determined by the percentage to be about 20 . Since the wind speed database is ten years long, it means that each month contains 10 sets of data. To fully cover the historical data, each set of random sampling is set twice.

\subsubsection{Calculation of Capacity Shortage}

The curves of the active power output by the simulated wind power and the active power absorbed by the auxiliary machine are superimposed on a histogram, as shown in Figure 2 .

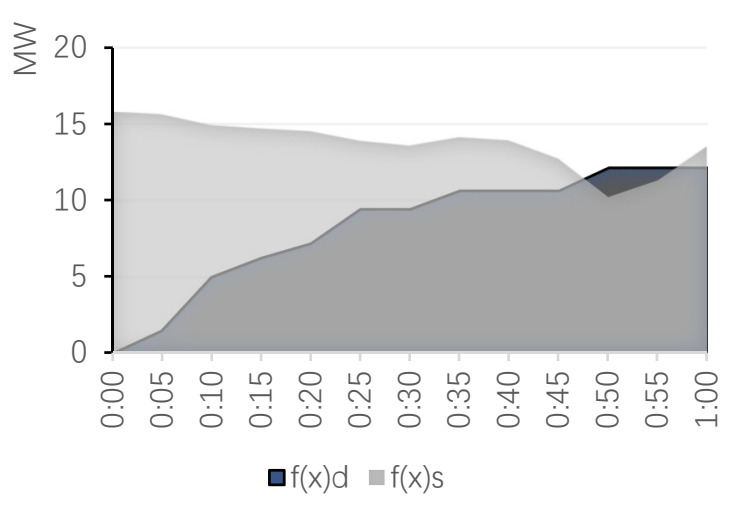

Figure 2 Supply and demand curve

The difference between the two curves will fluctuate positively and negatively as the wind power output is affected by the environment. When the difference is positive, it means that the power output of the wind power is short, and the integral of the difference over time is the shortfall of the load demand. From the difference, the expression of the deficit capacity can be obtained as:

$$
p=f(x)_{d}-f(x)_{s},(p>0)
$$

In the formula: $f(x)_{d}$ is the demand function, and $f(x)_{s}$ is the supply function. According to the definition of electricity, it generally refers to the accumulation of power over time. Integrating $t$ from both ends of the above formula can get the expression of the shortfall electricity:

$$
E=\int_{t_{2}}^{t_{1}}\left[f(x)_{d}-f(x)_{s}\right] d t \text {, and } f(x)_{d}-f(x)_{s}>0
$$

After 10 sets of randomly sampled time series wind speeds are imported into the simulation, MATLAB calculates the shortfall electricity to obtain the value, and then the data is counted by the following method. The maximum value of each group of data is added and divided by the number of groups to get the average value as the reference shortfall electricity, which is the following formula:

$$
\bar{E}=\frac{\sum_{i=1}^{10} \operatorname{Max}_{i k}}{10},(k=1,2)
$$

Where $\operatorname{Max}_{i k}$ is the maximum value in each group. Since the maximum power shortage in each simulation is basically an instantaneous value, its capacity configuration can cope with most of the instantaneous wind speed drops. Therefore, the lack of power will also calculate the maximum value in each simulation and then calculate the average value, which is the following formula:

$$
\bar{p}=\frac{p_{1}+p_{2}+\ldots+p_{20}}{20}=\frac{\sum_{i=1}^{20} p_{i}}{20}
$$

Where $P_{\mathrm{i}}$ is the maximum power difference in each simulation. 


\subsubsection{Capacity compensation calculation}

In the initial stage of the black start, energy storage needs to bear the start-up load of wind farm related equipment. In the calculation, the power consumption of a single wind power equipment is used as the base number multiplied by the scale of the wind farm as the compensation capacity.

Considering the efficiency of energy storage charging and discharging, it is necessary to set a certain amount of redundancy to compensate for the efficiency of actual engineering products. In order to maintain the energy storage activity, the charge of the energy storage device must be maintained in the range of $S O C_{\text {min }}<S O C_{t}<S O C_{\text {max }}$. When there is no black start requirement, the energy storage charge cannot exceed the maximum level, but the energy storage charge cannot be lower than its minimum value after supporting the black start.

\section{Case analysis}

An analysis of the black-start auxiliary power demand of a coal-fired thermal power plant with an installed capacity of $300 \mathrm{MW}$ in a thermal power station in Hulunbuir. Refer to Table 1 for the power requirements of the related auxiliary machines to obtain a total power requirement of approximately $12 \mathrm{MW}$.

Table 1 Starting time and power of auxiliary machinery of 300MW power plant

\begin{tabular}{lcl}
\hline Auxiliary equipment & Start Time & Power/kw \\
\hline Feed water pump & $0: 10$ & 3500 \\
other devices & $0: 05$ & 1500 \\
Circulating pump & $0: 15$ & 1250 \\
Condensate pump & $0: 20$ & 945 \\
Induced draft fan & $0: 25$ & 2240 \\
Blower & $0: 35$ & 1200 \\
Coal mill & $0: 50$ & 1500 \\
total capacity & & 12135 \\
\hline
\end{tabular}

According to the historical wind speed data of the wind farm, the annual average wind speed can be $4.4 \mathrm{~m} / \mathrm{s}$ from equation (1), and the initial scale of the wind farm is calculated from equation (2) to 18 units of $3 \mathrm{MW}$ wind power. The output of this wind farm is about $12 \mathrm{MW}$ at an average annual wind speed of $4.4 \mathrm{~m} / \mathrm{s}$. According to formula (5), the time series wind speed data of this wind field from 2010 to 2019 are statistically analyzed and its seasonal index is shown in Fig. 3, and the wind speed index in January is the lowest.

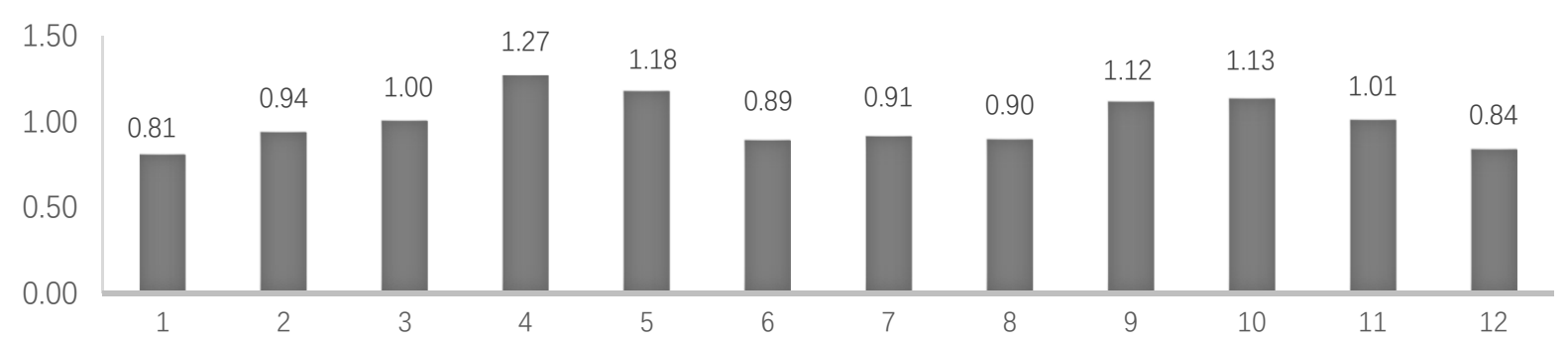

Figure 3 Monthly seasonal index statistics from 2010 to 2019

Based on the above calculations, build a wind farm with the same capacity on PSCAD. For the wind speed data that the model needs to input, please select the month with the lowest seasonal index as the sampling interval of the time series wind speed data. From the 10 sets of wind speed databases from 2010 to 2019 , the time series wind speed data obtained through two random samplings for each set are imported into the PSCD wind farm simulation model. One set of data is shown in Figure 4:

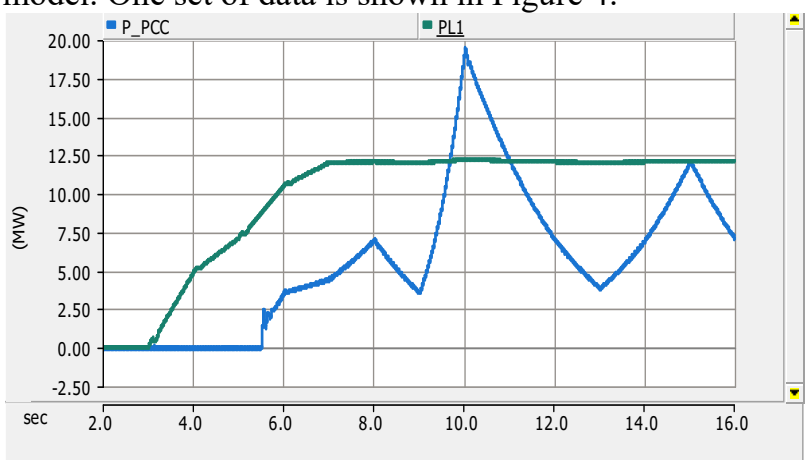

Figure 4. PSCAD wind power output power and load power demand comparison curve

The first 3 seconds of the simulation is the time to establish the bus voltage of the wind power system. The black start starts after 3 seconds and the time corresponding to the wind speed is equivalent in the simulation model. Import the curve data into MATLAB to calculate the power shortage, as shown in Figure 5.

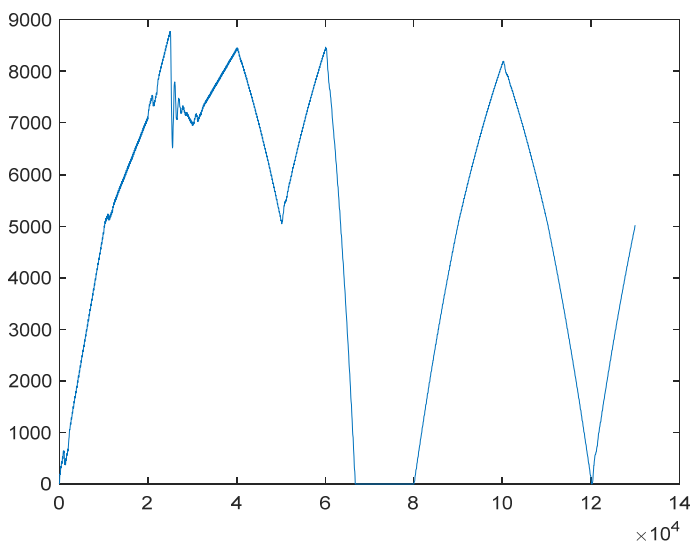

Figure 5. Power shortage curve

The power shortage value calculated in each simulation is calculated by the formula (8) to obtain the reference value. The maximum value of the power 
shortage curve in the current wind speed environment can be obtained from Figure 5, and the power capacity range of its energy storage and inverter equipment is determined by the statistics of formula (9). The calculated reference value is injected into the PSCAD wind storage black start model, and its energy storage power output characteristics follow the power shortage control of the factory bus. Randomly select 10 sets of data from the historical wind speed database to verify the success rate of the black start.

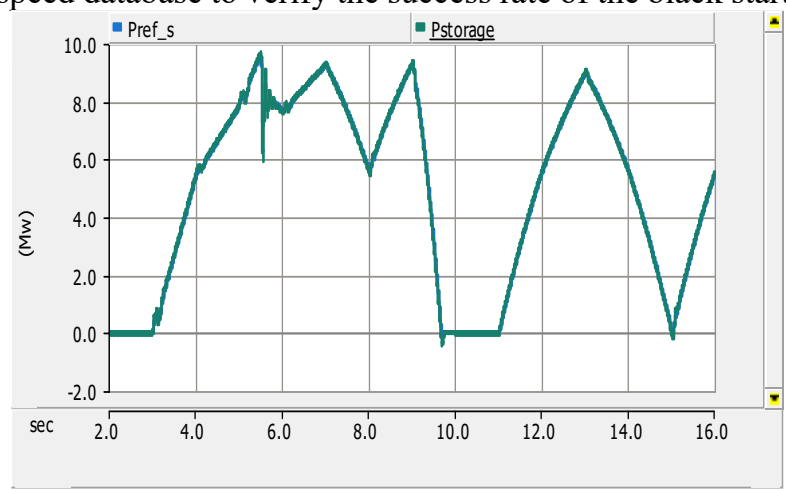

Figure 6. Energy storage power output curve

Figure 6 shows the response of energy storage to the power shortage in the system under a set of wind speeds. The power output of the wind storage system meets the load power demand under this wind condition as shown in Figure 7.

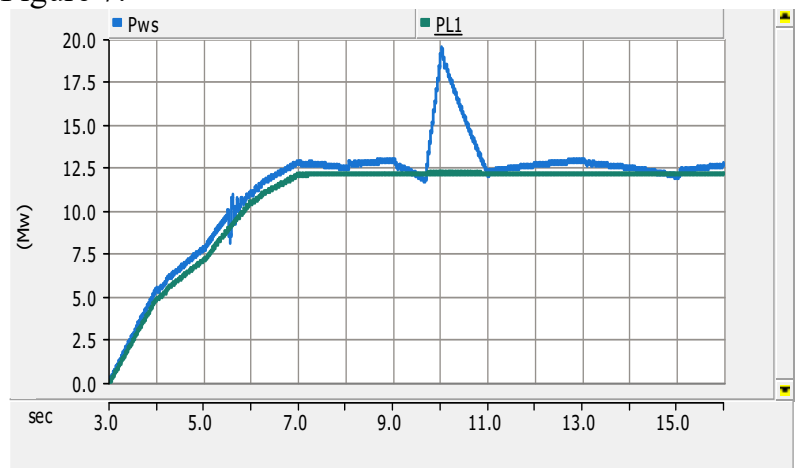

Figure 7. Comparison of wind storage active power output and load active power demand

If the wind farm needs to start automatically in the early stage of the black start, its power is usually provided by the energy storage system. The case uses a single $3 \mathrm{MW}$ wind power, referring to its product parameters, and it can be known that its related auxiliary equipment has a capacity of $35 \mathrm{KVA}$. This value is multiplied by the scale of the wind farm to obtain a capacity value of 648KVA. Compensation for energy storage capacity should refer to this value. Later, in actual engineering, additional energy storage redundancy can be added according to reliability requirements to cope with some extreme working conditions.

\section{Conclusion}

Based on the power demand of the plant and the historical wind speed data of the pre-selected wind farm, a calculation method for the energy storage capacity configuration of the wind storage system for black start purposes is proposed. This method is based on the historical wind speed data, and the month with the lowest seasonal index is used as the measurement benchmark for energy storage capacity, and the lack of capacity of auxiliary equipment can be obtained by processing the simulation image. In actual projects, the impact of the charging and discharging efficiency of energy storage products on the actual capacity of energy storage equipment requires reference to product parameters to leave redundancy. It is verified through simulation that the above-mentioned capacity configuration value scheme can effectively cope with the black start of the wind farm under most wind speed environments and is economical.

\section{References}

1. Cheng Shijun. Optimal configuration and operation research of wind farm energy storage system [D]; North China Electric Power University, 2014.

2. XIU X, TANG W, LI J, et al. Comprehensive Evaluation Technology of Energy Storage Configuration Based on Analytic Hierarchy Process [J]. Dianli Xitong Zidonghua/Automation of Electric Power Systems, 2018, 42(11): 72-8

3. Sun Yu, BOLLEN M, AULT G. Reliability analysis of distribution system with distributed power generation in island state. Power grid technology [J]. 2008, 32(23): 77-81.

4. Chen Zhigang. Optimization calculation method for energy storage capacity of large wind farms based on CFD technology [D]; Shanghai Jiao Tong University, 2015.

5. Mi Zengqiang, Sun Chaoyang, Liu Liqing, et al. Configuration method of battery energy storage system when energy storage wind farm is used as black start power source Electrical measurement and instrumentation[J]. 2018, 55(12): 20-6+ 124. 